\title{
Plasma-graft Anhydride on Carbon Fiber and its Effect on Composite Properties
}

\author{
Ya-Nan $\mathrm{YI}^{\mathrm{a}}$, Cai-Qiong ZHANG ${ }^{\mathrm{a}}$, Jing LONG ${ }^{\mathrm{a}}$, Lin ZHONG ${ }^{\mathrm{a}}$ \\ and Huan-Xia ZHANG ${ }^{*}$ a
}

\author{
a.College of Materials and Textile Engineering, Jiaxing University, Jiaxing \\ 314001, China
}

Correspondence to: Huan-Xia Zhang; *e-mail: zhanghuanxia818@163.com

Keywords: plasma grafting polymerization, DBD, rough, FSC test, interface

\begin{abstract}
The deposition of coatings on the surface of carbon fiber will be helpful to their applications. However, they are unsuitable to be deposited because of their low surface free energies, poor wettability and poor adhesions. The objective of this work is to modify carbon fibers by different methods of plasma grafting polymerization. The chemical and physical changes induced by the treatments on carbon fibers surface can be examined by using contact angle measurements and X-ray photoelectron spectroscopy (XPS). The interfacial adhesion of CF/EP composites is analyzed by a single fiber composite (SFC) for filament fragmentation test. The contact angles of the plasma-treated samples are visibly reduced compared to the untreated samples. The results of XPS reveal that the carbon fibers modified with the plasma grafting methods show a significant increase in oxygen-containing groups, such as $\mathrm{C}-\mathrm{O}, \mathrm{C}=\mathrm{O}$ and $\mathrm{O}-\mathrm{C}=\mathrm{O}$. The results of SFC tests show that the treated carbon fibers composites could possess excellent interfacial properties with mixed resins. These results demonstrate that the surfaces of the carbon fibers samples are more active, more hydrophilic and rougher after appropriate plasma grafting disposal.
\end{abstract}

\section{Introduction}

That carbon fiber reinforced polymer composites have been used widely in aerospace, marine and automobile industries during the past few decades (1960 onwards) due to their good engineering properties such as high specific strength and stiffness, lower density, high fatigue endurance, high damping and low thermal coefficient (in fiber direction), etc [1]. The mechanical properties of carbon fibers reinforced polymer composites are mainly controlled by the interfacial properties between the fiber and matrix even though they are also affected by the original nature of the fiber and matrix resin. [2]. However, as reinforcements for manufacturing high performance composite materials, carbon fibers have poor hygroscopicity and adsorption with most polymers which because carbon fiber (CF) surface is non-polar and compound of highly crystallized graphitic basal planes with inert structure

As a result, the interfacial bonding strength between the fibers and polymer matrices is low, and excellent mechanical performance of composites cannot be received [3-5]. Carbon fibers, without any surface treatment, cause a weak adhesion to the matrix resin. In order to improve the interfacial bonding intensities between the fiber and matrix, many surface treatment techniques were developed, including chemical method, $\gamma$-ray radiation, electrochemically oxidation, plasma-graft treatment, ozone $\left(\mathrm{O}_{3}\right)$ treatment, etc [6-7]. Many of these methods have the drawbacks of high energy consumption, time-consuming, environmental pollution and, in most cases, are accompanied by a decrease in fiber strength [8]. Plasma-graft polymerization method 
is relatively more convenient, more environmentally friendly, with less impact on the fiber bulk properties [9].

The surface treatment of carbon fibers was investigated with selected monomers via plasma-graft polymerization, and it was found that have significant improvements in both the interlaminar shear strength and flexural strength values of carbon fiber/epoxy composites. Plasma-graft polymerization method is an effective technique for the surface modification of polymers with plasma because the functional groups desired are able to be definitely introduced on the surface [2].

In this work it is shown how to obtain strong adhesion between a carbon fiber and an epoxy resin through the formation of covalent bonds. Anhydride groups are introduced onto the surface of the fiber by means of plasma-graft polymerization and then are reacted with the epoxy resin containing curing agent to form covalent bonds. The adhesive property between the grafted fiber and the epoxy resin is assessed by single fiber fragment test.

\section{Experiment}

\section{Materials and Surface Treatment}

The carbon fibers used in this study were PAN-based T300SC produced by Toray. The average diameter of these carbon fibers was approximately $7 \mu \mathrm{m}$, and typical tensile modulus and strength were about $240 \mathrm{GPa}$ and $3.5 \mathrm{GPa}$, respectively. Prior to use, the carbon fibers were cleaned in a Soxhlet extractor with acetone for $24 \mathrm{~h}$ to remove the size on the fiber surface. The epoxy matrix system for preparation of the SFC specimens was RIM 145 provided by Hexion Specialty Chemicals Inc. The graft polymerization was maleic anhydride solution, which dissolved in acetone with different concentrations $0.01 \mathrm{~mol} / \mathrm{L}, 0.03 \mathrm{~mol} / \mathrm{L}, 0.05 \mathrm{~mol} / \mathrm{L}$ and $0.07 \mathrm{~mol} / \mathrm{L}$.

The atmospheric pressure plasma treatments were performed using a DBD operating in argon. Two circular copper electrodes (diameter $=50 \mathrm{~mm}$ ) were placed in the DBD plasma configuration. Each electrode was covered with a ceramic plate with thickness of $2.2 \mathrm{~mm}$ and its area of $90 * 90 \mathrm{~mm}^{2}$. Before starting the experiments, samples were placed on the lower ceramic plate. When discharged, a large number of dense filamentary micro discharges generated from upper electrode and bombarded the surface of samples.

In this paper, it mainly studies two different plasma-graft polymerization methods. One is "A" method, the carbon fibers $40 \mathrm{~mm}$ long were immersed into $50 \mathrm{ml}$ of maleic anhydride solution for $2 \mathrm{~h}$, and then dried in vacuum at $100^{\circ} \mathrm{C}$, the dried carbon fibers were irradiated for $90 \mathrm{~s}$ with a plasma that were generated at discharge powers of 20-30W in an argon atmosphere, finally rinsed the carbon fibers with pure water under ultrasonic irradiation and dried $1 \mathrm{~h}$ in vacuum at $100^{\circ} \mathrm{C}$. Another method is " $\mathrm{B}$ ", the carbon fibers were irradiated for $90 \mathrm{~s}$ with a plasma that were generated at discharge powers of 20-30 W in an argon atmosphere, and then immersed for $2 \mathrm{~h}$ in 50 $\mathrm{ml}$ maleic anhydride solution. The plasma-grafted carbon fibers were washed with pure water under ultrasonic irradiation and then dried $1 \mathrm{~h}$ in vacuum at $100^{\circ} \mathrm{C}$.

\section{Characterization}

\section{Fiber Surface Morphology Analysis}

AFM (Multimode NanoscopeIIIa, Digital Instruments, USA) was used for investigation of the surface topology of untreated and treated carbon fibers. Topographical images were obtained in a contact mode. The roughness analysis of the surfaces was carried out from the images of a $5 \mu \mathrm{m} \times 5 \mu \mathrm{m}$ area. The root mean square 
roughness curve profile (RMS), the mean value of the surface relative to the center plane (mean roughness, $\mathrm{Ra}$ ), and the difference in height between the highest and lowest points on the surface relative to the mean plane (Rmax) were measured as functions of different treatment methods.

\section{Single Filament Tensile Tests}

The influence of atmospheric pressure plasma grafting treatment on the tensile strength of carbon fibers was determined by single filament tensile tests using an XQ-2 single fiber tensile tester (Shanghai Lipu Research Institute, Shanghai, China) equipped with a $2 \mathrm{~N}$ load cell. The tensile strength of the carbon fibers was measured at $10 \mathrm{~mm}$ gage lengths at a rate of $0.01 \mathrm{~mm} / \mathrm{s}$. Each sample contained at least 30 specimens. The two-parameter Weibull distribution function was adapted to evaluate the injury probability of the carbon fibers $[10,11]$.

\section{Contact Angle Measurements}

The surface free energy and contact angles of carbon fibers were measured by a DCAT II contact angle measuring device (Dtaphysics Instruments, Filderstadt, Germany). The fundamental tenet was that contact angles of test liquids on the fibers were measured by the modfied Wilhelmy technique using a microbalance. Distilled water and Ethylene glycol were used as test liquids.

\section{Evaluation of Interfacial Shear Strength[12]}

The SFC test method, originally developed from the early work of Kelly and Tyson [13], can provide abundant statistical information, such as interfacial failure modes and interfacial shear strength (IFSS) value, using only a few specimens [14]. Each test specimen for the fragmentation test consists of a single carbon fiber embedded in a matrix along the tensile loading direction. When the fiber's axial stress reaches its tensile strength, the fiber breaks and the tensile stress at the failed ends reduces to zero. The higher the strain, the more fiber breakages will occur.

In this study, samples for SFC tests were strained by Electronic Universal Tester. The samples were strained in steps of approximately $0.3 \%$ until no further breakage occurred. The ultimate fragment lengths were obtained from the digital images captured by a CCD camera and a computer digital imaging system. The diameters of the individual fibers were also measured and recorded using the microscope for calculation of IFSS.

\section{Results and Discussion}

\section{Fiber Surface Morphology Analysis}

The topographies of the untreated and the plasma graft treated carbon fibers were examined by AFM and shown in Fig. 1.
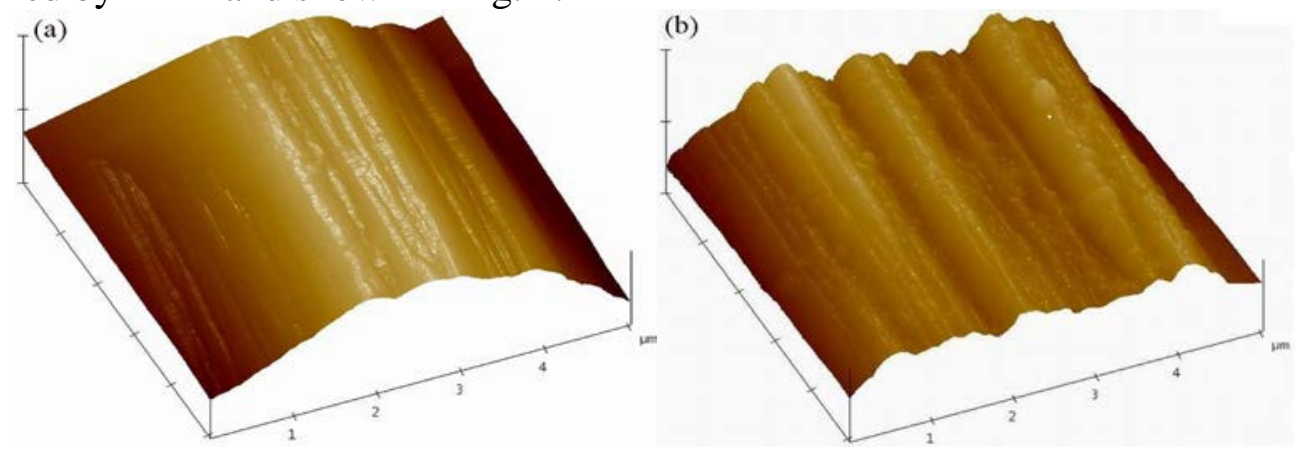


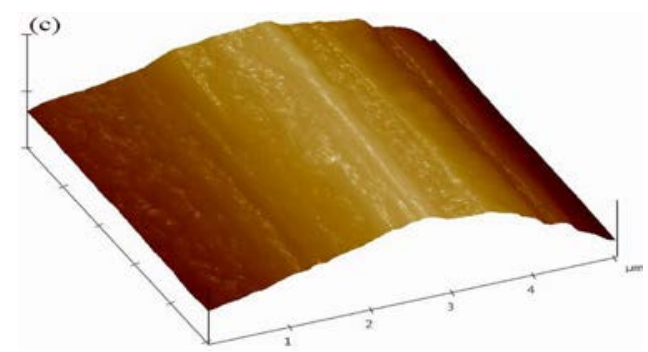

Fig.1 AFM micrographs of carbon fiber with different treatment methods: a reference,b 0.05A,c 0.05B

Well-defined streaks parallel to the fiber axis were observed before plasma grafting and the surface of the fiber was uneven. The streaks became unclear after plasma grafting. The surface roughness analysis data obtained by AFM software are listed in Table 1 . The surface roughness of the carbon fibers increased with plasma grafting treatment, and the roughness analysis dates split few differences with $0.05 \mathrm{~A}$ and 0.05B. The increase in surface roughness of plasma grafting treated carbon fibers should improve the interfacial bonding strength between the carbon fiber and matrix because a rougher surface morphology of the fiber led to a higher degree of mechanical interlocking in the interface region.

The surface of the carbon fiber without surface treatment is smooth, and with little or no groove. While after plasma treatment on carbon fiber surface is etched with obvious axial grooves,and the groove height analysis shows that $0.05 \mathrm{~B}$ height than 0.05A height.It means that the surface of carbon fiber after plasma treatment can effectively increase the surface roughness which improve the mechanical binding between carbon fiber and epoxy resin .

Table1 Roughness parameters of carbon fibers measured by AFM on images of $5 \mu \mathrm{m}$ $\times 5 \mu \mathrm{m}$

\begin{tabular}{lccc}
\hline Treatment voltage (kv) & Reference & $0.05 \mathrm{~A}$ & $0.05 \mathrm{~B}$ \\
\hline Root mean square roughness, RMS (nm) & 315 & 480 & 478 \\
Mean roughness, Ra (nm) & 273 & 414 & 418 \\
Maximum height, Rmax (nm) & 1326 & 1956 & 1839 \\
\hline
\end{tabular}

\section{Tensile strength of Carbon fibers}

To analyze the effects of the plasma treatment on the strength of carbon fiber, Weibull distribution was introduced. Set $\ln \ln [1 /(1-\mathrm{F})]$ as $\mathrm{Y}$ axis, and $\ln \sigma$ as $\mathrm{X}$ axis. All the Weibull plots and linear fitted lines were showed in Fig.2. 

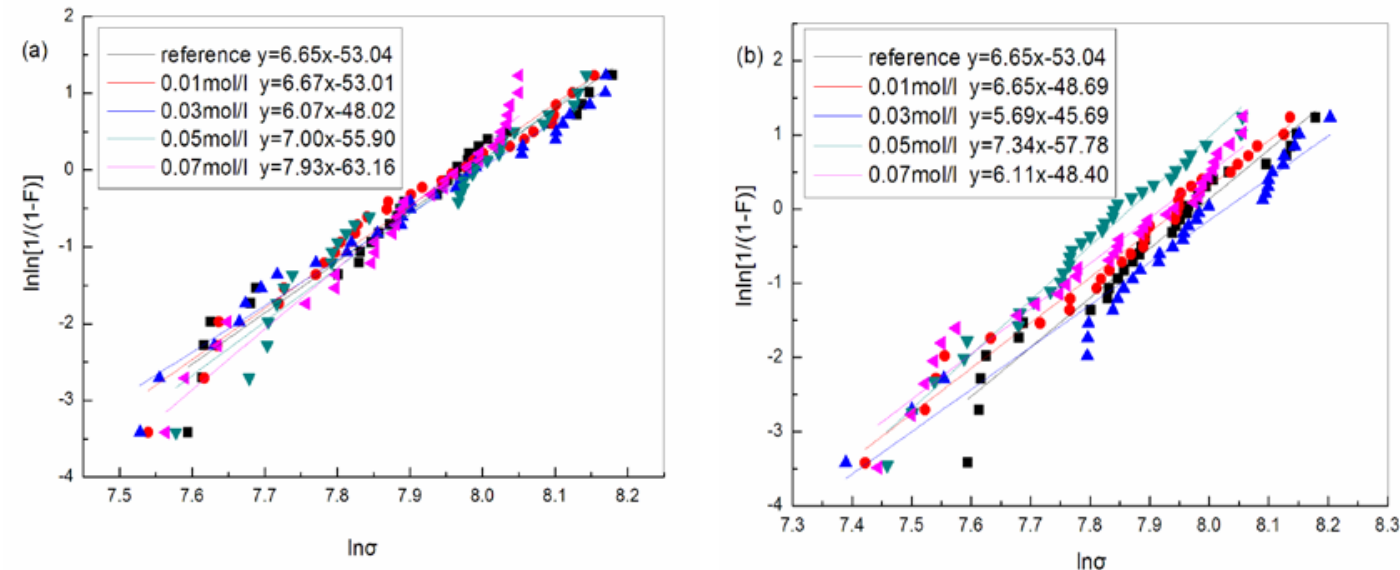

Fig.2 Weibull plots and linear fittings for carbon fiber with different plasma grafting treatment: A B

And it can be observed that the lines were fitted very well. The shape parameter $\rho$ and scale parameter $\sigma_{0}$ can be calculated from the slope and intercept of the fitted line. Shape parameter $\rho$ characterized for the distribution of the results, the smaller the value, the more reliable and intensive the results. Scale parameter signified the strength of the fiber. As we can see from Table. 2 that the shape parameters are all above 5.7, which indicates that the dispersion of the results is small, and also the plasma grafting treatment seems have marginally effects on the distribution of the strengths. As for the strength value, we can observe from Fig. 3 that the strength have not changed very much before or after the treatment. The strength increased about 140 $\mathrm{MPa}$ by the treatment of $0.03 \mathrm{~B}$. However, the strength generally decreased after treatment. It is noticed that the grafting solution cover and eliminate the small flaws of the fiber surface by the plasma grafting treatment, and leading to an increasing of the strength. On the other hand, severe etching may cause the expansion of the flaws, and further pull down the strength.

Table 2 Weibull scale and shape parameters for single carbon fiber tensile strengths.

\begin{tabular}{ccc}
\hline Samples & Scale parameter $\sigma_{0}$ & Shape parameter $\beta$ \\
\hline reference & 2922.43 & 6.65 \\
$0.01 \mathrm{~A}$ & 2893.59 & 6.67 \\
$0.03 \mathrm{~A}$ & 2730.06 & 6.07 \\
$0.05 \mathrm{~A}$ & 2928.87 & 7.00 \\
$0.07 \mathrm{~A}$ & 2867.58 & 7.93 \\
$0.01 \mathrm{~B}$ & 2836.55 & 6.13 \\
$0.03 \mathrm{~B}$ & 3062.74 & 5.69 \\
$0.05 \mathrm{~B}$ & 2611.14 & 7.34 \\
$0.07 \mathrm{~B}$ & 2751.68 & 6.11 \\
\hline
\end{tabular}

\section{Contact angles of carbon fibers}

The contact angles of untreated and plasma grafting treated carbon fibers against distilled water and ethylene glycol were presented in Fig. 3. The contact angles of carbon fiber with A method treatment are bodily smaller than B method. It also can be observed that the contact angle of the carbon fibers decreased with increasing concentration of maleic anhydride solution for B method. It decreased with increasing 
concentration to $0.05 \mathrm{~mol} / \mathrm{l}$ for A method, further increasing concentration to $0.07 \mathrm{~mol} / \mathrm{l}$ that the contact angle increased significantly. These results indicated that the surface of carbon fiber became more hydrophilic after plasma grafting treatment and increased concentration could not significantly further increase hydrophilicity of the carbon fiber surfaces.

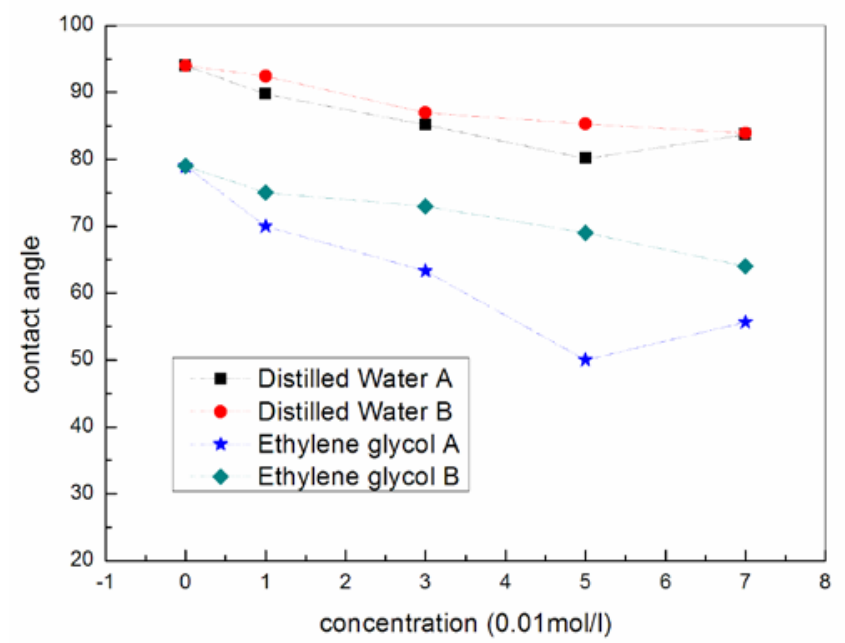

Fig.3 The change of contact angle with different plasma grafting treatment

\section{Interfacial shear strength between carbon fibers fibers and epoxy resin}

Table 3 Calculation of IFSS for carbon fiber/ epoxy resin interface.

\begin{tabular}{ccccc}
\hline Samples & $\begin{array}{c}\text { Critical fragment } \\
\text { length, } l_{c}(\mu \mathrm{m})\end{array}$ & $\begin{array}{c}\text { Tensile strength at } \\
l_{c}, \sigma_{f}(\mathrm{Mpa})\end{array}$ & IFSS, $\tau \quad$ (Mpa) & Increment(\%) \\
\hline reference & 0.50 & 4302.22 & 30.12 & 5 \\
0.01A & 0.50 & 4533.61 & 31.74 & 22 \\
0.03A & 0.43 & 4576.28 & 36.84 & 38 \\
0.05A & 0.39 & 4650.96 & 41.51 & 25 \\
0.07A & 0.40 & 4302.48 & 37.65 & 4 \\
0.01B & 0.51 & 4606.75 & 31.44 & 17 \\
0.03B & 0.51 & 5160.66 & 35.22 & 18 \\
0.05B & 0.40 & 4047.50 & 35.42 & 20 \\
0.07B & 0.44 & 4579.88 & 36.07 & \\
\hline
\end{tabular}

According to the above analysis, different treated methods can change the surface chemical composition and surface energy of the carbon fibers, which might have effect on the carbon fiber/epoxy interfacial adhesion. The interfacial shear strength (IFSS) of carbon fiber/epoxy was measured by SFC test. As can be seen from Table3, the IFSS of carbon fiber/epoxy increase with treatment concentration increasing to $0.05 \mathrm{~mol} / \mathrm{l}$ for A method. The effect of plasma grafting treatment on IFSS is researched that has two main factors. The activated groups on the surface of carbon fiber were increased after treatment before $0.05 \mathrm{~A}$ by XPS, which lead to the surface free energy of carbon fibers increased notability. The increased surface energy of carbon fibers made a 38\% increase in the adhesion between carbon fiber and epoxy resin. On the other hand, with even higher solution concentration, the carbon fibers surface was covered by grafting solution, which leads to decrease the interfacial adhesion between the fibers and the resin. As a result, the interface adhesion increase 
of the carbon fiber/epoxy composites was mainly due to increment of surface activated groups before $0.05 \mathrm{~A}$. However, with the increase the solution concentration for B method, the IFSS of carbon fiber/epoxy resin interface steadily increased to 36.07 Mpa.

\section{Conclusion}

The different plasma grafting treatment methods have different effect on the performance of carbon fiber and its composites. The surface roughness of the carbon fibers increased with different treated methods. The plasma grafting treatment improve obviously the surface energies of the carbon fibers, and with increasing grafting concentration for treatment, the $\mathrm{C}-\mathrm{C}$ concentration reduces whereas activated group concentrations increase, especially before the 0.05A. The single fiber strength of the plasma grafting treatment changes slightly than untreated sample. The IFSS of carbon fiber/epoxy increase with different treatment methods, especially 0.05A. At the same time, it is shown that the increase of interface adhesion was mainly due to increment of surface activated groups. And above all, the performance of carbon fiber treated with method A has reasonable improvement compared with method B.

\section{Acknowledgements}

The authors wound like to acknowledge the financial supported by the College students' Science and Technology Innovation Project of Zhejiang Province ( No. 2015R417010).

\section{References}

[1] S.P. Sandeep, K. Tarun, M.D. Yogesh, Compos. Struct., 84,114 (2008).

[2] J.P. Hou, X.D. Zhou and X.G. Zhou, Polymer-Plastics Technology and Engineering. 50, 260 (2011).

[3] K. Ma, Applied Surface Science, 257,3824 (2011).

[4] Z. W. Xu, Y. D. Huang and C.H. Zhang, Compos. Sci. Technol.,67, 3261 (2007).

[5] S. J. Park, and B. J. Kim, Sci. Eng. , A 408 , 269 (2005).

[6] B. Xu, X. S. Wang, and Y. Lu, Appl. Surf. Sci. ,253 , 2695 (2006).

[7] L. G. Tang, and J. L. Kardos, Polymer Composites, 18, 100 (1997).

[8] Z.W. Xu, X.Q. Wu and Y. Sun, J. Appl. Polym. Sci. ,108, 1887 (2008).

[9] A. Bismarck, D. Righter and C. Wuertz, J. Adhes. , 73, 19 (2000).

[10]An F, Lu CX, Li YH, Guo JH, Lu XX, Lu HB, et al. Preparation and characterization of carbon nanotube-hybridized carbon fiber to reinforce epoxy composite.Mater Des 2012;33:197 - 202.

[11] Rahman MM, Zainuddin S, Hosur MV, Malone JE, Salam MBA, Kumar A, et al.Improvements in mechanical and thermo-mechanical properties of e-glass/epoxy composites using amino functionalized MWCNTs. Compos Struct 2012;94(8):2397 406.

[12] H. X. Liu and Y. Z. Gu, Polymer Composites, 33,716(2012). 
[13] Vautard F, Fioux P, Vidal Lc, Siffer F, Roucoules V, Schultz J, et al. Use of plasma polymerization to improve adhesion strength in carbon fiber composites cured by electron beam. ACS Appl Mater Interfaces 2014;6(3):1662 - 74.

[14] Zaldivar RJ, Nokes JP, Kim HI. The effect of surface treatment on graphite nanoplatelets used in fiber reinforced composites. J Appl Polym Sci 2014;131(6):39994 . 